\title{
Effects of interval training on quality of life and cardiometabolic risk markers in older adults: a randomized controlled trial
}

This article was published in the following Dove Press journal:

Clinical Interventions in Aging

\author{
Marcel Ballin (1D ${ }^{1,2}$ \\ Emmy Lundberg (iD) ${ }^{1,2}$ \\ Niklas Sörlén ${ }^{1,2}$ \\ Peter Nordström ${ }^{\prime}$ \\ Andreas Hult (iD) ${ }^{2}$ \\ Anna Nordström (iD ${ }^{2,3}$ \\ 'Department of Community Medicine \\ and Rehabilitation, Unit of Geriatric \\ Medicine, Umeå University, Umeå, \\ Sweden; ${ }^{2}$ Department of Public Health \\ and Clinical Medicine, Section of \\ Sustainable Health, Umeå University, \\ Umeå, Sweden; ${ }^{3}$ Department of Sport \\ Science, School of Sport Sciences, The \\ Arctic University of Norway, Tromsø, \\ Norway
}

Purpose: To explore the effects of 10 weeks of progressive vigorous interval training as a single intervention on health-related quality of life (HRQoL) and cardiometabolic risk markers in centrally obese 70 -year-old individuals.

Participants and methods: A randomized controlled trial (ClinicalTrials.gov registration no. NCT03450655) including seventy-seven community-dwelling 70-year-old men and women with central obesity defined as $>1 \mathrm{~kg}$ visceral adipose tissue for women and $>$ $2 \mathrm{~kg}$ for men. Participants randomized to the intervention group were offered a 10 -week progressive vigorous interval training program performed three times per week. Control subjects were asked to maintain their daily living and routines throughout the trial. All participants in both groups had received tailored lifestyle recommendations focused on diet and physical activity at one occasion within 12 months prior to trial initiation. Prespecified outcome measures included: changes in HRQoL using the Short Form Health Survey Questionnaire (SF-36), blood pressure; resting heart rate (HR) and blood lipids. All analyses were conducted on an intention-to-treat basis.

Results: The intervention resulted in significant effects on the SF-36 mental component summary (MCS) score and the mental health $(\mathrm{MH})$ subscale $(P<0.05$ for both), when compared to the control group. Specifically, the intervention group increased their MCS score by 6.3 points $(95 \%$ confidence interval $[\mathrm{CI}]=0.3-12.3)$ and their $\mathrm{MH}$ score by 6.0 points $(95 \% \mathrm{CI}=1.7-10.4)$ compared to the control group. Moreover, significant effects were seen on resting HR, total cholesterol and LDL-cholesterol $(P<0.05$ for all).

Conclusion: It was shown that 10 weeks of vigorous interval training as a single intervention was sufficient to improve mental aspects of HRQoL in older individuals with central obesity, which is a critical aspect of healthy ageing. Positive effects were seen also on cardiometabolic risk markers.

Keywords: exercise, ageing, perceived health, blood lipids

\section{Introduction}

Human lifespan is rapidly increasing with the number of people $\geq 60$ expected to reach two billion by the year 2050. ${ }^{1}$ While ageing leads to an increased risk for cardiovascular disease $(\mathrm{CVD})^{2}$ which is the leading cause of mortality and morbidity, ${ }^{3}$ it is further distressing that many are also physically inactive to a greater extent than younger people ${ }^{4,5}$ and a large proportion experience obesity. ${ }^{6}$ These factors further increase the risk of CVD, ${ }^{7-10}$ however given their modifiable nature they may be the target of interventions. Therefore, interventions seeking to
Correspondence: Anna Nordström Department of Public Health and Clinica Medicine, Section of Sustainable Health, Umeå University, Umeå 90185 , Sweden Tel +46706064249

Email anna.h.nordstrom@umu.se 
promote physical activity in obese older adults and act on associated risk factors with CVD, including hypertension and dyslipidemia ${ }^{11-13}$ are important to study.

Even so, the importance of perceived health during ageing must not be forgotten as the ageing process is multidimensional and means facing a diversity of opportunities and difficulties. Health-related quality of life (HRQoL) is a construct which includes physical- and mental domains of health, ${ }^{14,15}$ which are important parts of a successful ageing. ${ }^{16}$ Low and declining HRQoL has been suggested to predict cardiovascular and all-cause mortality $^{17,18}$ and is associated also with obesity. ${ }^{19}$ To this end, interventions should, apart from targeting cardiometabolic risk factors, also aim to improve perceived aspects of health especially in obese older populations.

Previous studies indicate that exercise for older adults may improve cardiometabolic risk markers including high blood pressure and abnormal blood lipids. ${ }^{20-23}$ Although it seems as if exercise can improve also HRQoL in older adults, ${ }^{24}$ the evidence on the effects in obese older adults and the influence exercise intensity is limited. There are some indications that higher intensities may be favorable for improving HrQOL, ${ }^{25}$ and one previous study found positive effects from a one-year exercise intervention on HRQoL in obese older adults. ${ }^{26}$ However, the effects of short-term intensive exercise in older adults with central obesity has, to the best of our knowledge, not yet been studied. Therefore, the aim of this randomized controlled trial was to investigate if 10 weeks of vigorous interval training as a single intervention was sufficient to improve HRQoL and cardiometabolic risk markers in 70-year-old individuals with central obesity.

\section{Participants and methods Study design}

The present study was a randomized controlled trial (ClinicalTrials.gov registration number NCT03450655) aiming to investigate the effects of the current training program on a wide range of health-parameters. The primary outcome of the registered trial was visceral adipose tissue (VAT) and these findings have been published previously. ${ }^{27}$ For the present paper, the aim was to investigate the effects of the intervention on secondary prespecified outcomes including HRQoL and cardiometabolic risk markers. Specifically, the effect of the intervention was defined as the mean difference in changes on the outcomes from baseline to post-intervention between the study groups. The trial took place at a university hospital research clinic in Umeå, Sweden and was conducted in accordance with the World Medical Association's Declaration of Helsinki. Its design, methods and informed consent were granted permission by the regional research ethical review board of Umeå (Dnr 2017-521-31). Prior to the start of the trial, all participants were given both verbal- and written information about the trial before giving their written informed consent to participate in the trial. All participants were made aware of their possibility to voluntarily terminate their participation at any time. Reporting of the paper was performed according to CONSORT guidelines. ${ }^{28}$

\section{Participants}

Community-dwelling participants were selected and recruited between January 2018 and February 2018 from the ongoing population-based, primary prevention study, the Healthy Ageing Initiative (HAI). The HAI-cohort study has been described more thoroughly elsewhere, ${ }^{29}$ but in short, upon turning 70, all residents within Umeå municipality in northern Sweden are invited for a free health investigation aiming to explore and prevent future risk for developing chronic diseases. As part of their participation in HAI, the entire study sample had received tailored health recommendations with focus on diet and physical activity within 12 months prior to this trial.

\section{Inclusion and exclusion criteria}

To be included in the present trial, participants were required to have at least $1000 \mathrm{~g}$ and $2000 \mathrm{~g}$ of VAT, for women and men respectively, measured using a Lunar iDXA device with the CoreScan application (GE Healthcare Lunar, Madison, WI, USA) as described previously. ${ }^{27}$ The rationale for these values were that they have been shown to correspond to the cut-off points defining central obesity by waist circumference ( $>88 \mathrm{~cm}$ for women and $>102 \mathrm{~cm}$ for $\mathrm{men}^{30}$ ) for $2675 \mathrm{HAI}-$ participants. $^{27}$

Individuals with conditions that contraindicated training or affected the ability to perform the training program were excluded. The exclusion criteria were formalized as: physical disability affecting the ability to perform the exercises; heart failure or severe degenerative condition; myocardial infarction or stroke in the previous 12 months; heart condition that would worsen with aerobic exercise, eg angina pectoris; and systolic blood pressure (SBP) > $175 \mathrm{mmHg}$ and $(\mathrm{DBP})>100 \mathrm{mmHg}$. In addition, individuals had to pass a resting electrocardiogram (ECG; 
Schiller Cardiovit CS-6/12; Schiller AG, Switzerland). Any uncertainty that arose during baseline measurement regarding ECG results or contraindicated conditions was discussed with the physicians in charge of the trial (AN and $\mathrm{PN}$ ).

\section{Power calculation}

Statistical power and estimation of required sample size was based on the initial primary outcome for the current trial, VAT. Data on VAT mass from 2675 participants within the HAI-study were retrieved. The power calculation was performed using G*Power version 3.0.10 $0^{31}$ and performed separately for men and women. It was shown that a total of 33 men or 45 women per group, with somewhere in between that range for mixed groups, would provide $80 \%$ power to detect a $20 \%$ reduction in VAT, with the alpha level set to 0.05 .

\section{Randomization}

After completing the baseline assessments, individuals were then randomly assigned to either an intervention group or a control group, using 80 opaque sealed envelopes with notes of "Training" and "Control" (40 of each). To ensure allocation concealment, all envelopes were shuffled before each participant was given permission to draw an envelope and find out which group they were allocated to.

\section{Intervention}

The intervention consisted of a 10 -week weekly-progressive interval training program performed at a vigorous intensity in a group-setting under supervision from two fitness instructors with bachelor's degrees in sports medicine (MB and EL). Training sessions were based at a university hospital research clinic and performed three times per week in groups of 8-10 participants. Each session was preceded by a whole-body general warm-up and dynamic stretching for $10 \mathrm{~min}$, followed by vigorous interval training before ending with a 5-min relaxation and static stretching. In terms of the vigorous interval training, the work-to-rest ratio was prescribed to $40 / 20 \mathrm{~s}$. Initially, the vigorous interval training lasted for $18 \mathrm{~min}$ with a linear increment in duration throughout the intervention to a final maximum training duration of 36 mins. Thus, the total duration of the training sessions including warm-up, interval training and relaxation progressed from $33 \mathrm{~min}$ initially, towards $51 \mathrm{~min}$ by the later phase of the intervention. Volume increments were applied simultaneously for all participants in terms of progressively adding the number of working sets. The exercises used are presented in detail in Supplement 1. In short, they were dynamic exercises engaging large muscle groups that were designed to be easy-to-perform yet still allowing progression and regression depending on individual abilities and disabilities. Besides the use of suspension bands for some of the exercises and for balance support, no expensive gymequipment was used and no external resistance was applied during the exercises. Instead, each participants' own body weight served as their individual resistance.

Participants were advised to perform the intervals at a vigorous level of intensity. In older adults, vigorous intensity has previously been defined as being equivalent to 7 on a scale of $1-10$, which is characterized by rapid breathing and a substantial increase in HR. ${ }^{32}$ For the present study, the intensity was prescribed using a 10-level scale for the rate of perceived exertion (RPE) which was a modification of the Borg CR10 scale. To facilitate the use of the RPE-scale for the current population, the scale employed a color-coding and all levels on the scale were accompanied by a convenient description of the intensity characteristics of that specific level. Each of the six steps was labeled as follows; 1 Extremely light activity, 2-3 Light activity, 4-5 Moderate activity, 6-7 Vigorous activity, 8-9 Very vigorous activity, 10 Maximal exertion. Participants were encouraged to reach 6-7 during the intervals, which was characterized as challenging, although not completely exhausting, along with rapid breathing. As participants improved and progressed throughout the intervention, they were advised to exert an increased amount of effort by adjusting the tempo of the movements performed, as well as moving through a greater range of motion, in order to reach 6-7 on the scale. Prior to the start of the present study, a pilot-test of the training program was performed to assess the potential feasibility and acceptability of the training program. Please see Supplement 1 for a more detailed description. Control subjects were asked to maintain their daily living and routines throughout the trial.

\section{Assessment procedure}

Baseline- and outcome assessments were performed between 8 am and $5 \mathrm{pm}$. Outcome assessments were scheduled to be performed no more than 10 days following the last training session. Research nurses blinded to the intervention were in charge of the assessments during both occasions. All subjects had been instructed not to exert any intense physical activity 
nor consume alcohol the day prior to assessments, as well as being in a fasted state for a minimum of four hours. Participants self-reported medication, smoking, diabetes, and any history of CVD in terms of myocardial infarctions and stroke. Descriptive data on habitual physical activity were retrieved from the HAI-study where the participants were provided with a triaxial accelerometer (GT3X+; Actigraph, Pensacola, FL, USA) for seven consecutive days, as described previously in depth. ${ }^{29}$

\section{Outcome measures}

HRQoL was examined using the Short Form Health Survey Questionnaire (SF-36) which is a widely used, self-administered, health questionnaire comprised of 36 items that measures HRQoL on eight subscales. ${ }^{33,34}$ Each item is scored as $0-100$ where higher scores indicate better HRQoL. The score for each subscale is then produced by averaging all items for that subscale. The physical subscales include physical functioning $(\mathrm{PF})$; role limitations attributable to physical problems (RP); bodily pain (BP); and general health $(\mathrm{GH})$. The mental subscales include social functioning (SF); role limitations attributable to emotional problems (RE); vitality (VT); and mental health (MH). Physical- and mental component summary (PCS and MCS, respectively) scores are calculated by averaging the scores from the four physical- and the four mental subscales respectively.

Resting heart rate (HR), systolic blood pressure (SBP) and diastolic blood pressure (DBP) were measured using a digital automatic blood pressure device Omron M6 Comfort HEM-7221-E (Omron Healthcare, Kyoto, Japan) after a $15 \mathrm{~min}$ rest with the participant in a supine position. Two measurements were made whereof the lowest one was recorded and used in further analysis. Total cholesterol, (TC) high-density lipoprotein (HDL), low-density lipoprotein (LDL) and triglycerides (TG) were collected by venipuncture performed by research nurses after a minimum of four hours fasting. The blood samples were then sent to the accredited laboratory at the Department of Clinical Chemistry of Umeå University Hospital where they were analyzed.

\section{Statistical analysis}

Following visual inspection and test of skewness and kurtosis to assess normal distribution, either independent samples $t$-tests or Mann-Whitney $U$-tests were used to test for differences in continuous variables at baseline, while $\chi^{2}$-tests were used to test for categorical variables.
To analyze the effects of the intervention from baseline to post-intervention on the outcome measures within both groups separately, either paired $t$-tests or Wilcoxon signedrank tests were performed depending on whether data were deemed normally distributed or not. To compare the mean changes from baseline to post-intervention between the study groups a series of analysis of covariance (ANCOVA) with adjustments for baseline values were conducted. The significance level was set at $p \leq 0.05$ for the analyses on all outcome measures with the exception for the subscales constituting the MCS and PCS. For these, the original significance of $\leq 0.05$ was adjusted to $\leq 0.0125$ by Bonferroni correction to account for multiple testing. All statistical analyses were performed using SPSS software (v25.0; IBM Corp. Armonk, NY) and employed an intention-to-treat approach.

\section{Results \\ Participant flow, adherence and adverse events}

During the past 12 months, 427 individuals had participated in the HAI study. From these, 193 met the inclusion criterion and 103 were unavailable as they either did not respond to the invitation, declined participation or were subject to one or more of the exclusion criteria. This resulted in a study sample of $\mathrm{N}=90$ individuals eligible for study participation. During the baseline assessment $n=7$ were absent and thereby excluded, and $n=6$ failed to pass a resting ECG resulting in exclusion. Thus, a total of $\mathrm{N}=77$ individuals successfully completed baseline assessment and were randomized to an intervention group $(n=38)$ or to a control group $(n=39)$. Up until the time of follow-up assessment $n=3$ individuals from the control group voluntarily terminated their participation in the study. From the intervention group, $n=2$ individuals dropped out due to experienced discomfort as a result of the training and continuous illness that was unrelated to the exercise intervention. Additionally, during the course of the 10 -week intervention period five cases of adverse events related to the training were reported in terms of; Achilles tendinitis, lateral epicondylitis, knee bursitis, muscle strain and swelling in the metacarpophalangeal joint ( $\mathrm{n}=1$ each). Also, three participants reported incidents that were unrelated to the training in terms of; lumbago, wrist fracture and muscle strain ( $n=1$ each). It should be noted that neither of these events and conditions compromised the participants' ability to complete the follow-up assessment. The median attendance rate in the intervention group was $89 \%$ 
(interquartile range 80-96\%). An overview of the recruitment process and participant flow is presented in Figure 1.

\section{Baseline characteristics of the participants}

Participant characteristics are presented in Supplement 2. There were no significant differences between the two groups at baseline on any measures. The mean age of the participants was $70.7 \pm 0.2$ years, with a similar proportion of males and females (52\% female). Mean body mass index (BMI) was $29.2 \pm 3.3 \mathrm{~kg} / \mathrm{m}^{2}$. A total of $61 \%$ of the participants in the total cohort received hypertension medication, and $42 \%$ lipid lowering medication, at the start of the trial.

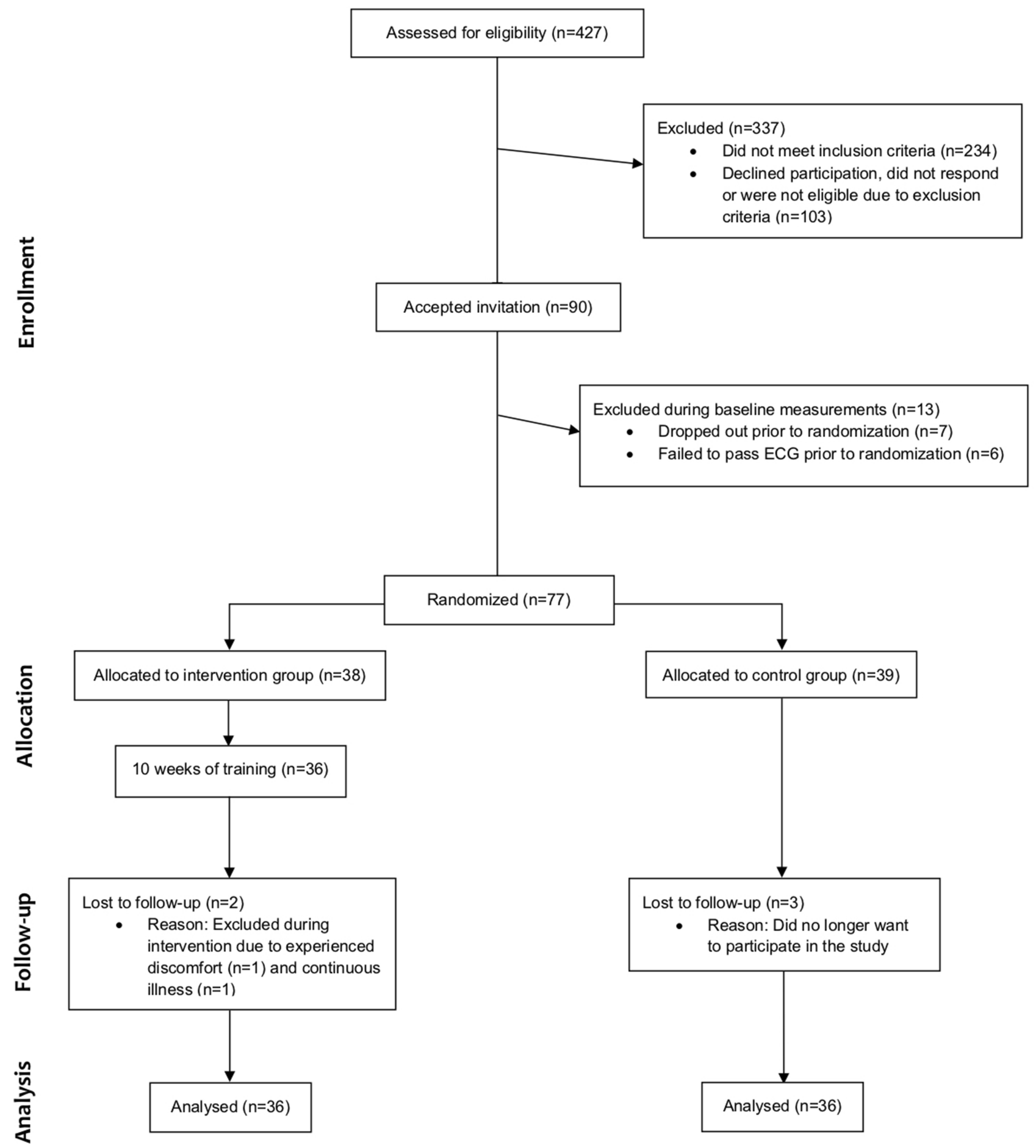

Figure I Study flow chart previously provided as supporting online information in Ballin et al $2019 .{ }^{27}$ 


\section{Effects of the intervention on health- related quality of life}

The ANCOVAs showed statistically significant differences between the study groups in mean change from baseline to post-intervention on the MCS, VT scale and MH scale before the Bonferroni correction in favor of the intervention group (Table 1, Figure 2). Specifically, the intervention group improved their MCS score by a mean of 6.3 points $(95 \%$ confidence interval $[\mathrm{CI}]=0.3-12.3, P=$ $0.040)$ and their $\mathrm{MH}$ score by 6.0 points $(95 \% \mathrm{CI}=1.7-$ $10.4, P=0.007)$, compared to the control group. The intervention group also improved their VT score by a mean of 6.0 points $(95 \% \mathrm{CI}=1.2-10.8, P=0.015)$ compared to the control group, although this was no longer considered statistically significant after the Bonferroni correction.

The analyses of changes within each study group separately showed significant improvements in the intervention group on the VT- and PF scales $(P<0.05$ for both) from baseline to post-intervention, although the effect on PF was not statistically significant after Bonferroni correction. Participants in the control group significantly decreased their score on the MH scale $(P<0.05)$ although not statistically significant after Bonferroni correction (Table 1).

\section{Effects of the intervention on blood pressure and resting heart rate}

The ANCOVAs showed statistically significant differences between the groups in mean change in resting HR from baseline to post-intervention, where the intervention group lowered their resting HR by 3.7 beats per minute (bpm) (95\% CI $=0.2-7.3, P=0.045)$ compared to the control group. There was no significant difference between the groups in change on BP, (Table 1, Figure 3) although participants in the intervention group significantly lowered SBP with $7.3 \pm 11.8 \mathrm{mmHg}$ and DBP with $4.2 \pm$ $6.8 \mathrm{mmHg}$ (both $P<0.001$ ).

\section{Effects of the intervention on lipids}

Looking at the effects on blood lipids the results from the ANCOVAs showed that the intervention group reduced TC by $0.35 \mathrm{mmol} / 1(95 \% \mathrm{CI}=0.08-0.62, P=0.012)$ and $\mathrm{LDL}$ by $0.27 \mathrm{mmol} / 1(95 \% \mathrm{CI}=0.06-0.49, P=0.014) \mathrm{com}-$ pared to the control group. These outcomes were also significantly improved within the intervention group itself $(P<0.05$ for both) (Table 1, Figure 3).

\section{Discussion}

The present randomized controlled trial showed that 10 weeks of vigorous interval training was sufficient to yield significant beneficial effects on mental aspects of HRQoL when compared to a control group. There were also favorable effects on cardiometabolic risk markers including TC, LDL and resting HR.

Research suggest that HRQoL is important for the individual's perception of a healthy ageing, as well as for increased longevity. ${ }^{16-18}$ Consequently, it is encouraging that the present intervention resulted in statistically significant and clinically meaningful improvements ${ }^{35}$ on the MCS as well as the MH subscale. This is in contrast to a previous meta-analysis ${ }^{24}$ which found no clear benefits. Improvements in HRQoL could be related to weight loss from the activity, as in a previous review. ${ }^{36}$ However, participants in our trial did not significantly lose weight. ${ }^{27}$ Our results are supported by another study ${ }^{37}$ which showed effects of exercise on HRQoL independently of weight-change. This is an important finding as while weight loss is a key component in obesity-therapy it is often accompanied by loss of vital lean body mass, which can have severe health consequences for older adults. ${ }^{38}$ The mechanisms that mediate the relationship between exercise and improved mental health is not clear, although it is hypothesized that one mechanism could be related to the secretion of endorphins and monoamines. ${ }^{39}$ However, psychological- and psychosocial factors such as self-efficacy and social interaction should also be considered, ${ }^{39,40}$ and the association between social engagement and HRQoL in older adults have previously been studied. ${ }^{41}$ To this end, we speculate that parts of the observed effects from the intervention on mental health may have been the results of participants experiencing an enhanced perceived ability to perform the training program throughout the intervention and appreciating being in a homogenous group where the participants encouraged and inspired each other.

While physical aspects of HRQoL are commonly improved following exercise, ${ }^{24}$ the present intervention did not manage to reproduce these findings, although the tendencies were in the direction of benefit. Two previous studies on overweight and obese older adults ${ }^{26,42}$ reported significant effects of exercise on these outcomes, however those interventions lasted between 6-8 months. Thus, the present 10week intervention was perhaps insufficient to achieve such effects, supported by a suggested dose-dependent association between exercise volume and improved HRQoL. ${ }^{37}$ As such, it is clearly interesting that the intervention nevertheless was sufficient to improve mental aspects of HRQoL Henceforth, 


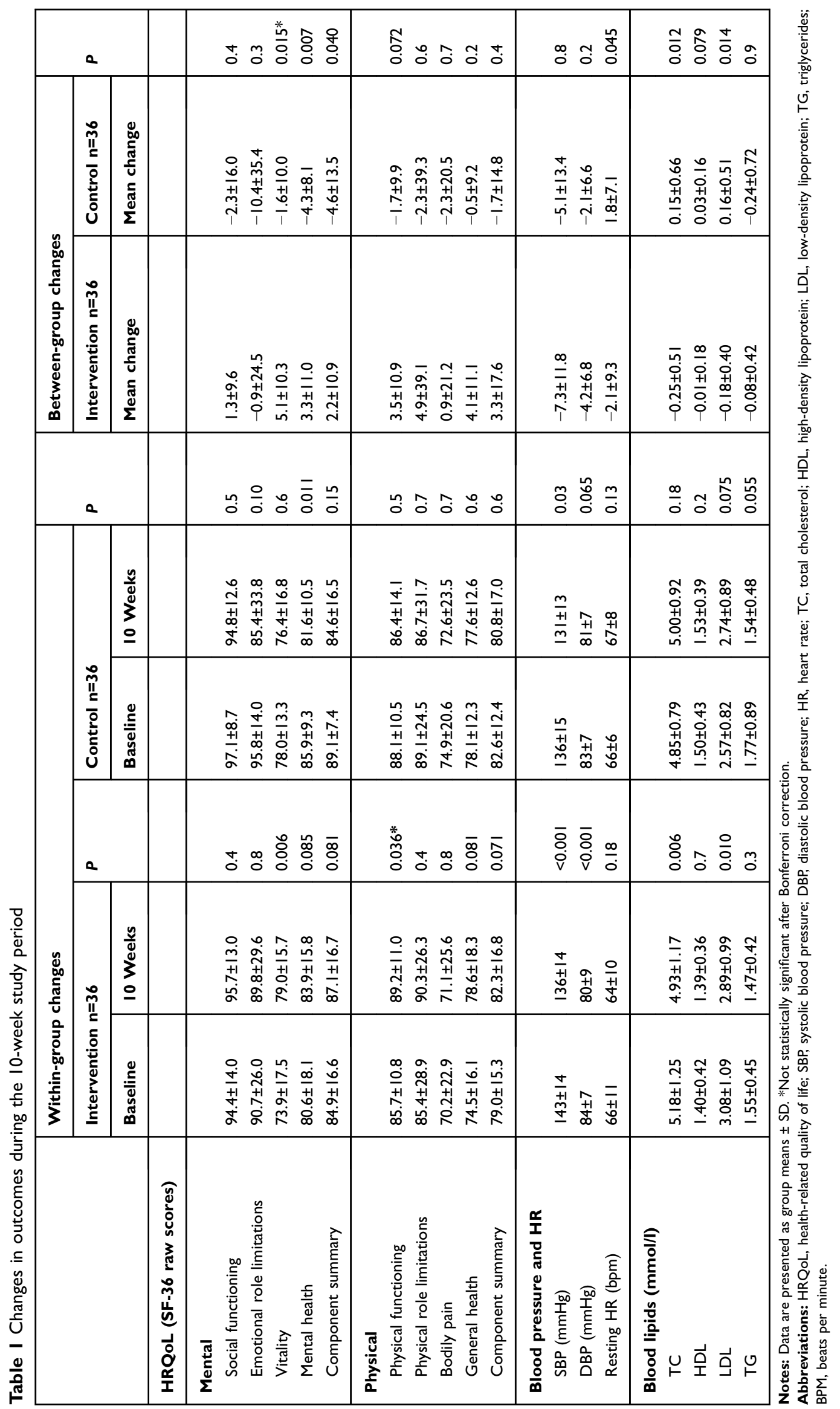




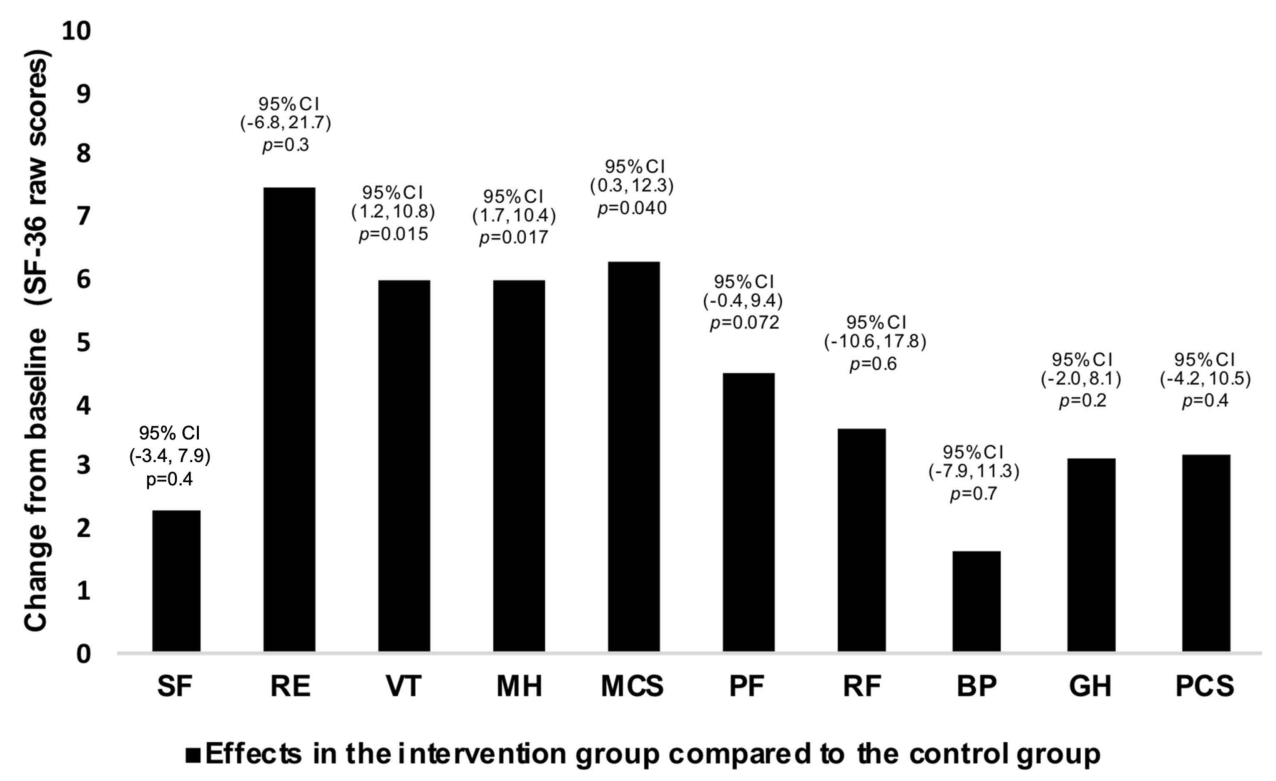

Figure 2 Mean changes and 95\% confidence intervals for SF-36 raw scores across all subscales and component summaries from baseline to follow-up for the intervention group compared to the control group. $P$-values are derived from ANCOVA on mean change from baseline to follow-up adjusted for baseline values.

Abbreviations: BP, bodily pain; $\mathrm{Cl}$, confidence interval; $\mathrm{GH}$, general health; MCS, mental component summary; MH, mental health; PCS, physical component summary; PF, physical functioning; RE, emotional role functioning; RF, physical role functioning; SF, social functioning; VT, vitality.

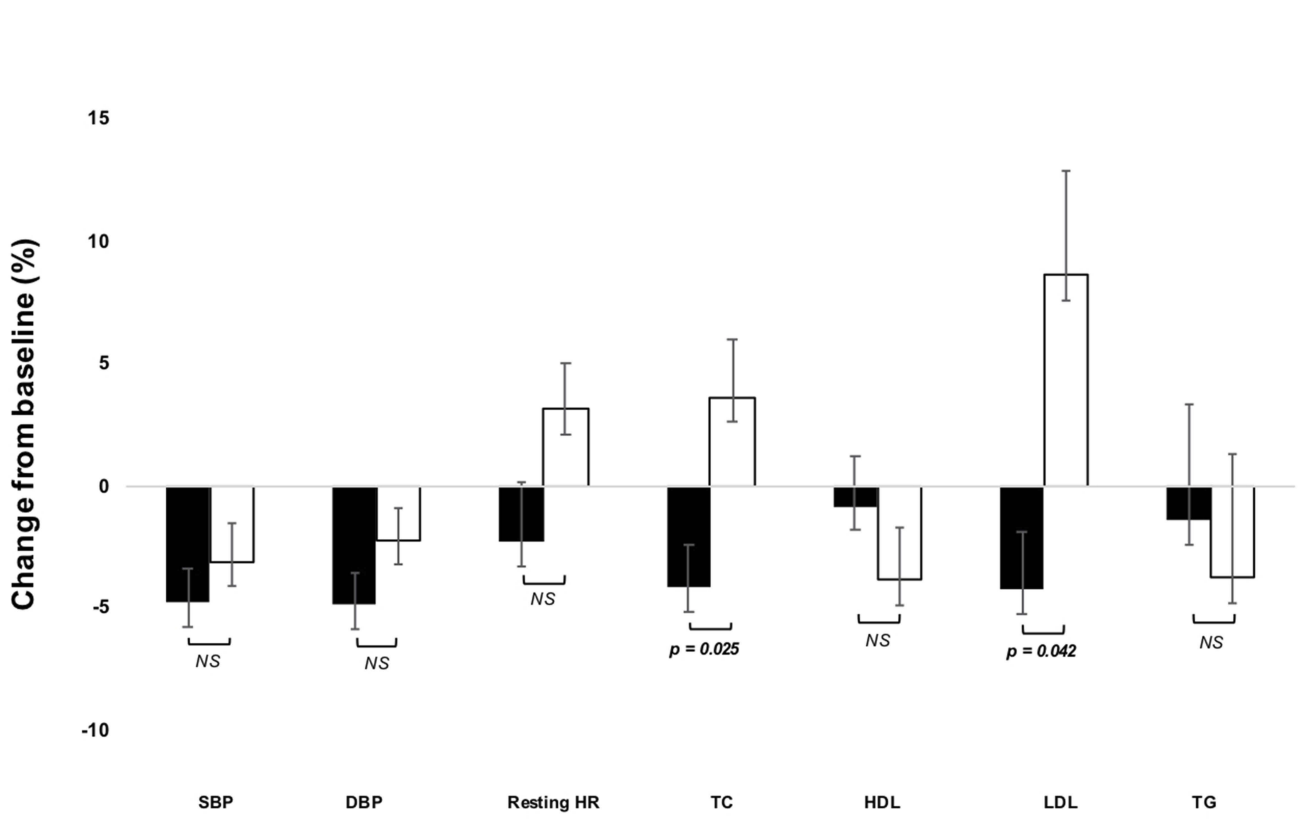

Figure 3 Mean percentage changes in cardiometabolic risk markers in the study groups following the 10-week intervention period. Error bars represent standard errors of the mean. $P$-values are derived from ANCOVAs on mean change from baseline to follow-up in percent adjusted for baseline values.

Abbreviations: DBP, diastolic blood pressure; HDL, high-density lipoprotein; HR, heart rate; LDL, low-density lipoprotein; SBP, systolic blood pressure; TC, total cholesterol; TG, triglycerides.

we encourage future studies to explore how manipulation of training volume may influence the outcome.

Due to the high prevalence of hypertension and its impact on risk of CVD and mortality, ${ }^{43}$ prevention and treatment of this condition is critical. Previous studies indicate that exercise have positive effects on blood pressure, ${ }^{44}$ which in some cases and populations are similar to those of antihypertensive medications. ${ }^{45}$ While evidence shows that older adults may achieve significant reductions in blood pressure following exercise, ${ }^{20,46}$ 
10 weeks of the present intervention was not sufficient to detect a statistically significant difference between the groups. Furthermore, elevated resting HR has been associated with a greater risk of developing hypertension and proposed as a predictor for $\mathrm{CVD}^{47}$ and cardiovascular mortality. ${ }^{48}$ It has previously been shown that exercise may lower resting HR by an average of six bpm in older adults, ${ }^{49}$ although this is commonly seen in studies exceeding 30 weeks. ${ }^{49}$ Thus, the length of our intervention may be a possible explanation to the seemingly smaller reductions in resting HR in the present study. Despite that the tendencies were favoring the intervention group and that larger reductions may potentially be seen if exercise is sustained over time, this warrants further confirmation before any conclusions can be drawn.

Given that abnormal lipids have proven to be a major risk factor for CVD, ${ }^{11,13}$ with exercise proposed as a beneficial method for improving lipid profile, ${ }^{50-52}$ our findings provide further support for the positive effects on TC and LDL, also in older populations. While previous research has shown that exercise can be beneficial also for improving HDL, ${ }^{53}$ this was not found in the present study, potentially caused by an insufficient training volume. ${ }^{52}$ Moreover, it has been suggested that individuals with a BMI $>28$, as in our population, are less prone to exerciseinduced effects on HDL, and that both weight- and body fat loss is required to improve lipid metabolism. ${ }^{53}$ Consequently, a combination of an exercise- and dietary intervention may yield larger effects on the lipid profile. Moreover, exercise has shown to result in positive effects on these outcomes in patients with diagnostic values of these variables ${ }^{54}$ and the fact that the current study population was not included based on diagnostic values of blood lipids could be another explanation to the findings. Future studies should therefore evaluate the effects of vigorous interval training in obese older adults with abnormal levels of blood lipids specifically in order to help bring more clarity to the matter.

Some limitations of this trial, including the sample size, must be considered. As previously mentioned, initial power calculations to determine an appropriate sample size were based on VAT, due to that being the primary outcome of the original trial. Thus, it is likely that the sample size deemed necessary to detect changes on VAT does not apply to detect changes on outcomes included in this present paper. We are also unable to conclude to what extent participants reached a vigorous intensity as the level of intensity was not objectively measured. The primary strengths of the present paper lie in the randomized design with blinded research personnel in charge of all assessments, and in the personalized training program resulting in an adherence that is to be considered high. ${ }^{55}$ The pilot test of the training session conducted in a reference group prior to the start of the study allowed fine tuning of the training program to fit the current population, and as a result, only a handful of easy-to-perform exercises were used. These could be performed without the use of expensive gym-equipment, yet still allowing both progression and individual adjustment to reduce the risk of injuries. Taken together, this may have promoted feelings of selfefficacy by ensuring that the participants felt that they could master the exercises, which in turn was reinforced by seeing their peers succeeding. ${ }^{56}$ The above-mentioned factors have previously also been identified as facilitators for engaging in exercise among older adults. ${ }^{57,58}$ Moreover, it is important to highlight the fact that there were relatively few adverse events, none of which lasted throughout the entire intervention period. Fear of injuries is a barrier to engage in exercise among older people, ${ }^{57}$ thus it is encouraging that the present study found vigorous interval training to be not only feasible and effective, but also safe to perform for older adults. Because of the minimal use of equipment, we believe that the training program may potentially be performed also in a home environment.

\section{Conclusion}

To conclude, this paper demonstrates that 10 weeks of vigorous interval training is sufficient to improve mental aspects of HRQoL in centrally obese older adults, with simultaneous effects also on LDL, TC and resting HR. HRQoL is a critical aspect of a healthy ageing, thus improvements of this component apart from objective measures is of great value. Nevertheless, more randomized trials are needed to confirm the present findings of improved mental health following exercise as well as comparing the effects between different exercise modalities, especially in obese older populations.

\section{Data sharing statement}

Individual participant data will not be available in accordance with The General Data Protection Regulation. The study protocol and statistical analysis plan is available at https://clinicaltrials.gov/ct2/show/NCT03450655. 


\section{Acknowledgment}

The authors would like to acknowledge research nurses Magnus Lindblom, David Lapveteläinen and Jonas Johansson for their work with data collection. We would also like to thank Monica Ahlenius for her work with participant recruitment. Finally, we are deeply grateful to all the participants who made this trial possible.

\section{Disclosure}

The authors report no conflicts of interest in this work.

\section{References}

1. United Nations, Department of Economic and Social Affairs, Population Division (2017). World Population Ageing 2017 Highlights (ST/ESA/SER. A/397).

2. North BJ, Sinclair DA. The intersection between aging and cardiovascular disease. Circ Res. 2012;110:1097-1108. doi:10.1161/ CIRCRESAHA.111.246876

3. Piepoli MF, Hoes AW, Agewall S, et al. 2016 European Guidelines on cardiovascular disease prevention in clinical practice: the Sixth Joint Task Force of the European Society of Cardiology and Other Societies on Cardiovascular Disease Prevention in Clinical Practice (constituted by representatives of 10 societies and by invited experts) Developed with the special contribution of the European Association for Cardiovascular Prevention \& Rehabilitation (EACPR). Eur Heart J. 2016;37:2315-2381. doi:10.1093/eurheartj/ehw106

4. Hallal PC, Andersen LB, Bull FC, et al. Global physical activity levels: surveillance progress, pitfalls, and prospects. Lancet. 2012;380:247-257. doi:10.1016/S0140-6736(12)60646-1

5. Dumith SC, Hallal PC, Reis RS, Kohl HW. Worldwide prevalence of physical inactivity and its association with human development index in 76 countries. Prev Med. 2011;53:24-28. doi:10.1016/j. ypmed.2011.02.017

6. Fakhouri THI, Ogden CL, Carroll MD, Kit BK, Flegal KM. Prevalence of Obesity among Older Adults in the United States, 2007-2010. US Department of Health and Human Services, Centers for Disease Control and ...; 2012.

7. World Health Organization. Global health risks: mortality and burden of disease attributable to selected major risks. https://apps.who. int/iris/handle/10665/44203. Accessed July 2, 2019.

8. Kenchaiah S, Evans JC, Levy D, et al. Obesity and the risk of heart failure. N Engl J Med. 2002;347:305-313. doi:10.1056/NEJMoa020245

9. Yusuf S, Hawken S, Ounpuu S, et al. Obesity and the risk of myocardial infarction in 27,000 participants from 52 countries: a case-control study. Lancet. 2005;366:1640-1649. doi:10.1016/S0140-6736(05)67663-5

10. Lee IM, Shiroma EJ, Lobelo F, et al. Effect of physical inactivity on major non-communicable diseases worldwide: an analysis of burden of disease and life expectancy. Lancet. 2012;380:219-229. doi:10.1016/S0140-6736(12)61031-9

11. Yusuf S, Hawken S, Ounpuu S, et al. Effect of potentially modifiable risk factors associated with myocardial infarction in 52 countries (the INTERHEART study): case-control study. Lancet. 2004;364:937952. doi:10.1016/S0140-6736(04)17018-9

12. Herrington W, Lacey B, Sherliker P, Armitage J, Lewington S. Epidemiology of atherosclerosis and the potential to reduce the global burden of atherothrombotic disease. Circ Res. 2016;118:535546. doi:10.1161/CIRCRESAHA.115.307611

13. Butler J, Rodondi N, Zhu Y, et al. Metabolic syndrome and the risk of cardiovascular disease in older adults. $\mathrm{J} \mathrm{Am}$ Coll Cardiol. 2006;47:1595-1602. doi:10.1016/j.jacc.2005.12.046
14. Testa MA, Simonson DC. Assessment of quality-of-life outcomes. NEngl J Med. 1996;334:835-840. doi:10.1056/NEJM199603283341306

15. Hickey A, Barker M, McGee H, O’Boyle C. Measuring healthrelated quality of life in older patient populations: a review of current approaches. Pharmacoeconomics. 2005;23:971-993. doi:10.2165/ 00019053-200523100-00002

16. Phelan EA, Anderson LA, Lacroix AZ, Larson EB. Older adults' views of "successful aging"- how do they compare with researchers' definitions? J Am Geriatr Soc. 2004;52:211-216. doi:10.1111/j.1532-5415.2004.52056.x

17. Myint PK, Luben RN, Surtees PG, et al. Relation between selfreported physical functional health and chronic disease mortality in men and women in the European prospective investigation into cancer (EPIC-Norfolk): a prospective population study. Ann Epidemiol. 2006;16:492-500. doi:10.1016/j.annepidem.2005.04.005

18. Otero-Rodríguez A, León-Muñoz LM, Balboa-Castillo T, Banegas JR, Rodríguez-Artalejo F, Guallar-Castillón P. Change in healthrelated quality of life as a predictor of mortality in the older adults. Qual Life Res. 2010;19:15-23. doi:10.1007/s11136-009-9561-4

19. Kolotkin RL, Meter K, Williams GR. Quality of life and obesity. Obes Rev. 2001;2:219-229.

20. Kelley GA, Sharpe Kelley K. Aerobic exercise and resting blood pressure in older adults: a meta-analytic review of randomized controlled trials. J Gerontol A Biol Sci Med Sci. 2001;56:M298-M303. doi:10.1093/gerona/56.5.m298

21. Herrod PJJ, Doleman B, Blackwell JEM, et al. Exercise and other nonpharmacological strategies to reduce blood pressure in older adults: a systematic review and meta-analysis. J Am Soc Hypertens. 2018;12:248-267. doi:10.1016/j.jash.2018.01.008

22. Kelley GA, Kelley KS, Tran ZV. Exercise, lipids, and lipoproteins in older adults: a meta-analysis. Prev Cardiol. 2005;8:206-214.

23. Halverstadt A, Phares DA, Wilund KR, Goldberg AP, Hagberg JM. Endurance exercise training raises high-density lipoprotein cholesterol and lowers small low-density lipoprotein and very low-density lipoprotein independent of body fat phenotypes in older men and women. Metabolism. 2007;56:444 450. doi:10.1016/j.metabol.2006.10.019

24. Kelley GA, Kelley KS, Hootman JM, Jones DL. Exercise and healthrelated quality of life in older community-dwelling adults: a metaanalysis of randomized controlled trials. J Appl Gerontol. 2009;28:369-394. doi:10.1177/0733464808327456

25. De Vries NM, Van Ravensberg CD, Hobbelen JSM, Rikkert MGMO, Staal JB, Nijhuis-Van der Sanden MWG. Effects of physical exercise therapy on mobility, physical functioning, physical activity and quality of life in community-dwelling older adults with impaired mobility, physical disability and/or multi-morbidity: a meta-analysis. Ageing Res Rev. 2012;11:136-149. doi:10.1016/j.arr.2011.11.002

26. Villareal DT, Chode S, Parimi N, et al. Weight loss, exercise, or both and physical function in obese older adults. $N \mathrm{Engl} \mathrm{J} \mathrm{Med}$. 2011;364:1218-1229. doi:10.1056/NEJMoa1008234

27. Ballin M, Lundberg E, Sorlen N, Nordstrom P, Hult A, Nordstrom A. Effects of interval training on visceral adipose tissue in centrally obese 70-year-old individuals: a randomized controlled trial. J Am Geriatr Soc. 2019;67:1625-1631. doi:10.1111/jgs.15919

28. Altman DG, Schulz KF, Moher D, et al. The revised CONSORT statement for reporting randomized trials: explanation and elaboration. Ann Intern Med. 2001;134:663-694. doi:10.7326/0003-4819-134-8-20010417000012

29. Johansson J, Nordstrom A, Nordstrom P. Objectively measured physical activity is associated with parameters of bone in 70-year-old men and women. Bone. 2015;81:72-79. doi:10.1016/j.bone.2015.07.001

30. National Cholesterol Education Program (NCEP) Expert Panel on Detection, Evaluation, and Treatment of High Blood Cholesterol in Adults (Adult Treatment Panel III). Third Report of the National Cholesterol Education Program (NCEP) Expert Panel on Detection, Evaluation, and Treatment of High Blood Cholesterol in Adults (Adult Treatment Panel III) final report. Circulation. 2002;106:3143-3421. 
31. Faul F, Erdfelder E, Lang AG, Buchner A. G*Power 3: a flexible statistical power analysis program for the social, behavioral, and biomedical sciences. Behav Res Methods. 2007;39:175-191.

32. Nelson ME, Rejeski WJ, Blair SN, et al. Physical activity and public health in older adults: recommendation from the American College of Sports Medicine and the American Heart Association. Circulation. 2007;116:1094-1105. doi:10.1161/CIRCULATIONAHA.107.185650

33. Ware JE, Sherbourne CD. The MOS 36-item short-form health survey (SF-36). I. Conceptual framework and item selection. Med Care. 1992;30:473-483.

34. Walters SJ, Munro JF, Brazier JE. Using the SF-36 with older adults: a cross-sectional community-based survey. Age Ageing. 2001;30:337-343. doi:10.1093/ageing/30.4.337

35. Samsa G, Edelman D, Rothman ML, Williams GR, Lipscomb J, Matchar D. Determining clinically important differences in health status measures: a general approach with illustration to the health utilities index mark II. Pharmacoeconomics. 1999;15:141-155. doi:10.2165/00019053-199915020-00003

36. Kroes M, Osei-Assibey G, Baker-Searle R, Huang J. Impact of weight change on quality of life in adults with overweight/obesity in the United States: a systematic review. Curr Med Res Opin. 2016;32:485-508. doi:10.1185/03007995.2015.1128403

37. Martin CK, Church TS, Thompson AM, Earnest CP, Blair SN. Exercise dose and quality of life: a randomized controlled trial. Arch Intern Med. 2009;169:269-278. doi:10.1001/archinternmed.2008.545

38. Waters DL, Ward AL, Villareal DT. Weight loss in obese adults 65 years and older: a review of the controversy. Exp Gerontol. 2013;48:1054-1061. doi:10.1016/j.exger.2013.02.005

39. Paluska SA, Schwenk TL. Physical activity and mental health. Sports Med. 2000;29:167-180. doi:10.2165/00007256-200029030-00003

40. Phillips SM, Wójcicki TR, McAuley E. Physical activity and quality of life in older adults: an 18-month panel analysis. Qual Life Res 2013;22:1647-1654. doi:10.1007/s11136-012-0319-z

41. Hajek A, Brettschneider C, Mallon T, et al. The impact of social engagement on health-related quality of life and depressive symptoms in old age - evidence from a multicenter prospective cohort study in Germany. Health Qual Life Outcomes. 2017;15:140. doi:10.1186/s12955-017-0715-8

42. Canuto Wanderley FA, Oliveira NL, Marques E, Moreira P, Oliveira J, Carvalho J. Aerobic versus resistance training effects on health-related quality of life, body composition, and function of older adults. $J \mathrm{Appl}$ Gerontol. 2015;34:NP143-NP165. doi:10.1177/0733464812468502

43. Kearney PM, Whelton M, Reynolds K, Muntner P, Whelton PK, He J. Global burden of hypertension: analysis of worldwide data. Lancet. 2005;365:217-223. doi:10.1016/S0140-6736(05)17741-1

44. Whelton PK, He J, Appel LJ, et al. Primary prevention of hypertension: clinical and public health advisory from The National High Blood Pressure Education Program. JAMA. 2002;288:1882-1888. doi:10.1001/jama.288.15.1882
45. Naci H, Salcher-Konrad M, Dias S, et al. How does exercise treatment compare with antihypertensive medications? A network meta-analysis of 391 randomised controlled trials assessing exercise and medication effects on systolic blood pressure. Br J Sports Med. 2019;53:859-869. doi:10.1136/bjsports-2018099921

46. Huang G, Shi X, Gibson CA, Huang SC, Coudret NA, Ehlman MC. Controlled aerobic exercise training reduces resting blood pressure in sedentary older adults. Blood Press. 2013;22:386-394. doi:10.3109/ 08037051.2013 .778003

47. Palatini P, Julius S. Elevated heart rate: a major risk factor for cardiovascular disease. Clin Exp Hypertens. 2004;26:637-644.

48. Zhang D, Shen X, Qi X. Resting heart rate and all-cause and cardiovascular mortality in the general population: a meta-analysis. CMAJ. 2016;188:E53-E63. doi:10.1503/cmaj.150535

49. Huang G, Shi X, Davis-Brezette JA, Osness WH. Resting heart rate changes after endurance training in older adults: a meta-analysis. Med Sci Sports Exerc. 2005;37:1381-1386.

50. Snowling NJ, Hopkins WG. Effects of different modes of exercise training on glucose control and risk factors for complications in type 2 diabetic patients: a meta-analysis. Diabetes Care. 2006;29:25182527. doi: $10.2337 / \mathrm{dc} 06-1317$

51. Lin X, Zhang X, Guo J, et al. Effects of exercise training on cardiorespiratory fitness and biomarkers of cardiometabolic health: a systematic review and meta-analysis of randomized controlled trials. $J$ Am Heart Assoc. 2015;4. doi:10.1161/JAHA.115.002014

52. Mann S, Beedie C, Jimenez A. Differential effects of aerobic exercise, resistance training and combined exercise modalities on cholesterol and the lipid profile: review, synthesis and recommendations. Sports Med. 2014;44:211-221. doi:10.1007/s40279-013-0110-5

53. Kodama S, Tanaka S, Saito K, et al. Effect of aerobic exercise training on serum levels of high-density lipoprotein cholesterol: a meta-analysis. Arch Intern Med. 2007;167:999-1008. doi:10.1001/ archinte.167.10.999

54. Ostman C, Smart NA, Morcos D, Duller A, Ridley W, Jewiss D. The effect of exercise training on clinical outcomes in patients with the metabolic syndrome: a systematic review and meta-analysis. Cardiovasc Diabetol. 2017;16:110. doi:10.1186/s12933-017-0624-5

55. Picorelli AM, Pereira LS, Pereira DS, Felício D, Sherrington C. Adherence to exercise programs for older people is influenced by program characteristics and personal factors: a systematic review. $J$ Physiother. 2014;60:151-156. doi:10.1016/j.jphys.2014.06.012

56. Bandura A. Health promotion by social cognitive means. Health Educ Behav. 2004;31:143-164. doi:10.1177/1090198104263660

57. Bethancourt HJ, Rosenberg DE, Beatty T, Arterburn DE. Barriers to and facilitators of physical activity program use among older adults. Clin Med Res. 2014;12:10-20. doi:10.3121/cmr.2013.1171

58. Schutzer KA, Graves BS. Barriers and motivations to exercise in older adults. Prev Med. 2004;39:1056-1061. doi:10.1016/j.ypmed.2004.04.003

Clinical Interventions in Aging

Dovepress

\section{Publish your work in this journal}

Clinical Interventions in Aging is an international, peer-reviewed journal focusing on evidence-based reports on the value or lack thereof of treatments intended to prevent or delay the onset of maladaptive correlates of aging in human beings. This journal is indexed on PubMed Central, MedLine, CAS, Scopus and the Elsevier

Bibliographic databases. The manuscript management system is completely online and includes a very quick and fair peer-review system, which is all easy to use. Visit http://www.dovepress.com/ testimonials.php to read real quotes from published authors. 